\title{
La conception des circuits hydrauliques et ses répercussions sur le génie civil des écluses
}

\section{Hydraulic circuit design and its effects on the civil engineering features of locks}

\author{
M. A. Petitjean \\ Ingénieur des Ponts et Chaussées au Service Technique Central \\ des Ports Maritimes et des Voies Navigables
}

Les évolutions qui sont retracées dans cet exposé ne concernent que les écluses des voies navigables à grand gabarit de France.

Il ne s'agit pas toujours d'évolutions très récentes, vu lo petit nombre d'ouvrages mis en service ces dernières années, mais plutôt d'un panorama des idées qui prévalent depuis 10 ans environ.

L'écluse est l'ouvrage classique de franchissement des chutes par les bateaux; après les "écluses simples" sont apparues au $\mathrm{XV}^{\mathrm{e}}$ siècle les "écluses à sas", dont le principe de fonctionnement reste inchangé, et qui sont les weules utilisées aujourd'hui en France.

Comme chacun le sait, l'écluse repose sur le "principe des vases communicants", le sas constituant un petit "vase" mis en communication avec un grand "vase", le bief amont ou le bief aval, dont le niveau est peu affecté par cette opération de remplissage ou de vidange du sas.

Le sas de l'écluse doit donc être dimensionné au plus juste pour que les quantités d'eau qui s'écoulent à chaque cycle, et les temps d'échange, soient les plus laibles possibles; cette contrainte hydraulique ainsi que la facilité d'amarrage des bateaux, imposent très généralement d'avoir un sas à bajoyers verticaux, qui jouent le rôle de murs de soutènement, dont les dimensions en plan déterminent celles du plus grand bateau pouvant circuler sur la voie.

Il est donc déjà clair que l'aspect général des écluses est étroitement conditionné par la conception de leur circuit hydraulique: en effet, un circuit permettant d'échanger des débits nettement plus importants sans agitation supplémentaire dans le sas pourrait permettre, sur des rivières à débit important, de réaliser des sas d'écluses qui seraient de véritables biefs courts à berges inclinées, éventuellement courbes en plan, que les bateaux parcoureraient pendant la durée des échanges d'eau.

Mais les contraintes hydrauliques marquent encore plus fortement la conception de la structure du sas, certaines conceptions, qui pourraient être économiques du seul point de vue "mur de soutènement", devant être rejetées car ne permettant pas de loger le circuit hydraulique.

\section{Conception du circuit hydraulique}

On considère que, sur une grande écluse (classes IV, V et VI), les temps d'échange d'eau ne doivent pas excéder $5 \mathrm{mn}$ par demi-cycle; il s'agit en fait d'un temps relativement faible par rapport à la durée du même demicycle (environ $30 \mathrm{mn}$ ), qui est surtout conditionnée par les durées d'entrée (environ $15 \mathrm{mn}$ ) et de sortie (environ $8 \mathrm{mn}$ ) des bateaux; mais c'est le seul sur lequel on puisse actuellement jouer, et de plus il représente psychologiquement l'équivalent d'un "feu rouge" routier.

\section{Systèmes d'alimentation et de vidange du sas}

Afin de respecter cette durée sans provoquer d'agitation excessive dans le sas, différents systèmes sont envisageables, selon l'importance de la chute rachetée par l'écluse :

1) jusqu'à environ $2 \mathrm{~m}$, une alimentation par les portes, très généralement réalisée par vantelles (vannes glissantes venant obturer des ouvertures ménagées dans les vantaux) ;

2) de $2 \mathrm{~m}$ à $5 \mathrm{~m}$, une alimentation par contoumement des têtes;

3) de $5 \mathrm{~m}$ à $12 \mathrm{~m}$, une alimentation par aqueducs larrons répartis le long du bajoyer (bajoyer épais); 


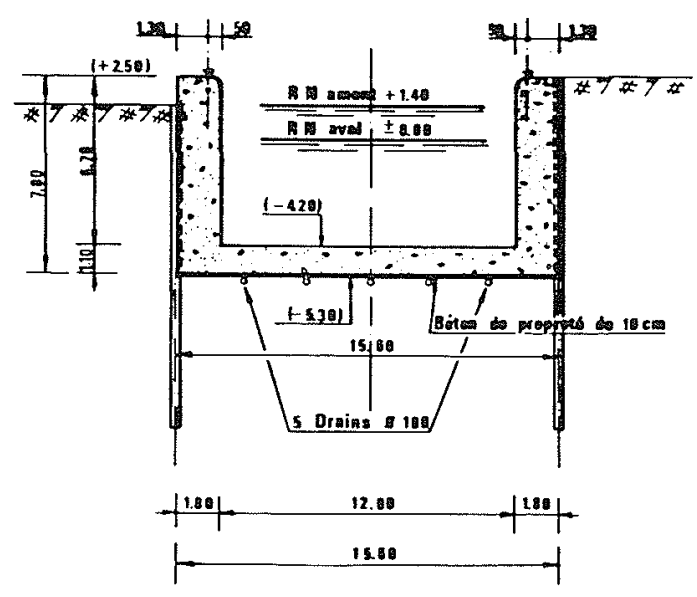

Figure 1. - Bajoyers en palplanches avec parement béton; alimentation par les portes (écluse de Venette; Oise; chute = $1,30 \mathrm{~m})$

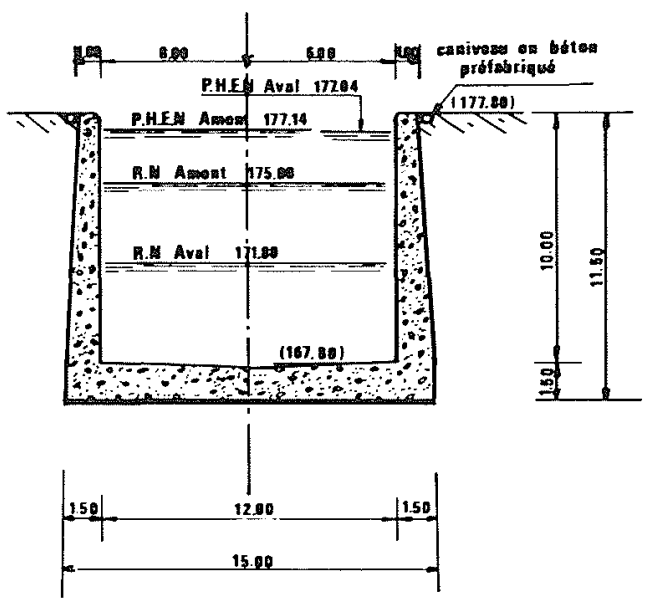

Figure 2. - Sas en $U$ en béton armé ; alimentation par contournement des têtes (écluse d'Ecuelles; Saône; chute $=3,20 \mathrm{~m}$ )

4) au-delà, une alimentation par des orifices de petite section répartis le long du radier (radier épais).

La hiérarchie des dispositifs énumérés ci-dessus repose sur un coût de réalisation croissant, s'accompagnant

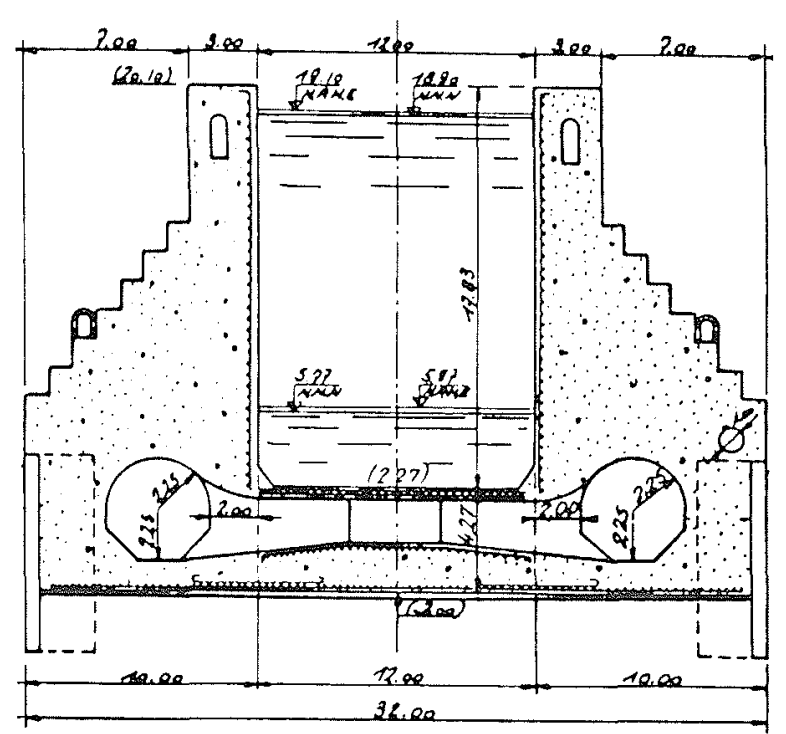

Figure 4. - Bajoyers autostables; alimentation par le radier (écluse des Fontinettes; canal Dunkerque; Valenciennes; chutes $=13 \mathrm{~m}$ )

d'une meilleure dissipation d'énergie dans le sas qui permet de prélever, et surtout d'injecter, dans le sas des débits croissants avec la chute en maintenant un confort de l'usager inchangé ; ainsi, à l'écluse St-Pierre, sur le Rhône, de $23 \mathrm{~m}$ de chute, le $4^{\mathrm{e}}$ dispositif permet une vitesse ascensionnelle des bateaux de $3,6 \mathrm{~m} / \mathrm{mn}$ soit un débit échangé de $130 \mathrm{~m}^{3} / \mathrm{s}$ environ.

Pour le premier système mentionné, il est souvent utile de supprimer le mur de chute de l'écluse, afin que les vantelles de la porte amont s'ouvrent au niveau le plus proche possible du radier; ceci conduit donc à augmenter la hauteur de la porte amont.

Pour le second système, on prévoit généralement une chambre de dissipation d'énergie au débouché dans le sas des conduites de contournement amont.

Pour le troisième système, une légère surprofondeur de l'écluse par rapport aux normes (qui prévoient dans

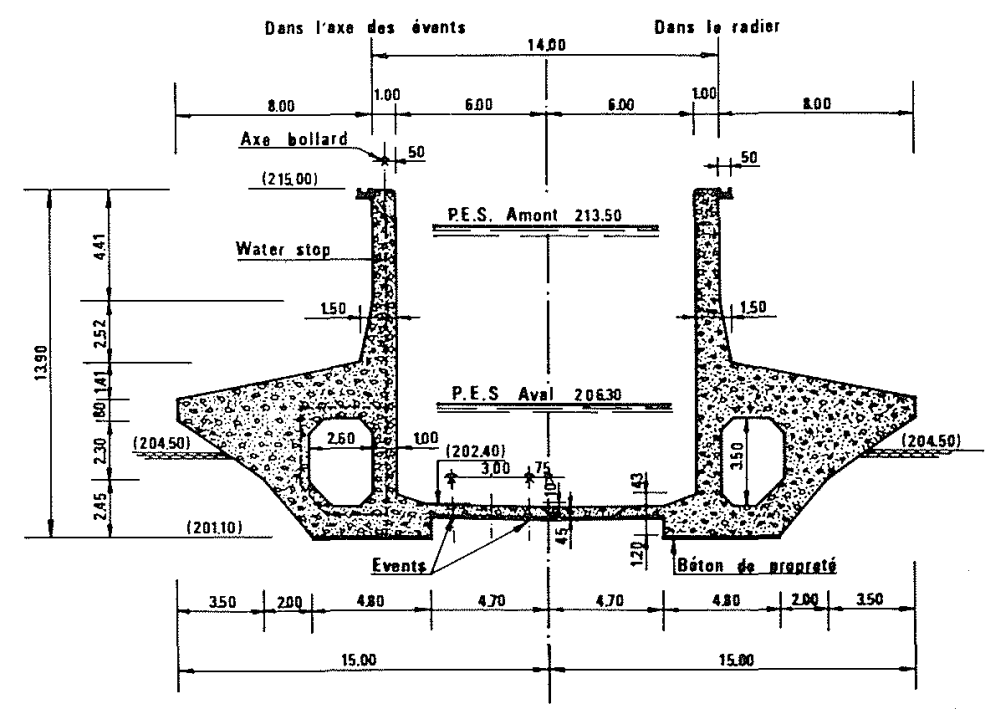

Figure 3. - Bajoyers autostables; alimentation par aqueducs larrons (ecluse de Villey-Le-Sec; Moselle; chute $=7,20 \mathrm{~m}$ ) 
l'écluse un pied de pilote de $1 \mathrm{~m}$ pour un enfoncement de $2,50 \mathrm{~m}$, et de $1,5 \mathrm{~m}$ pour un enfoncement de $3 \mathrm{~m}$ ) permet d'éviter que le jet des larrons ne puisse frapper directement la coque des bateaux; de plus, afin de limiter la vitesse d'arrivée de l'eau dans le sas, la section des larrons est choisie largement supérieure à celle de l'aqueduc d'alimentation, dans lequel l'eau s'écoule à des vitesses pouvant atteindre $4 \mathrm{~m} / \mathrm{s}$.

Pour le troisième comme pour le quatrième système, on s'assure également que le débit des différentes arrivées d'eau dans le sas est équilibré, afin d'y éviter la création d'ondes dangereuses pour l'amarrage des bateaux; pour cela, on peut disposer de deux conduites, l'une directe de chaque tête jusqu'au milieu du sas, où elle alimente la seconde, qui alimente à son tour le sas ; on peut aussi, plus simplement, avoir des orifices de section plus réduite à l'amont du sas qu'à l'aval, mais cette disposition n'est efficace qu'au remplissage.

Pour illustrer ce que nous annoncions plus haut sur linteraction entre alimentation en eau et conception de structure du sas, on voit donc par exemple qu'un bajoyer en palplanches métalliques habillées de béton ne pourra être retenu, lorsque l'alimentation peut être réalisée par aqueducs larrons, que si cette structure reste compétitive avec une alimentation par le radier, plus pénalisante au niveau du coût. Remarquons aussi à ce propos qu'il n'est en général pas nécessaire de prévoir la possibilité de mise à sec complète de l'écluse avec les deux premiers systèmes, mais que cela est souhaitable pour les deux derniers (opération d'entretien), ce qui a aussi une répercussion sur le dimensionnement des bajoyers.

\section{Répercussion sur les biefs}

Ayant ainsi résolu les problèmes hydrauliques concernant le sas de l'écluse, il convient de prendre garde aux phénomènes d'ondes et de vitesse en bief, où :

- les efforts horizontaux exercés sur un bateau de poids $W$ par une onde de pente $i$ s'élèvent à $W i$ (dans le sas de l'écluse, où le phénomène est beaucoup plus complexe, on a trouvé expérimentalement $2 \mathrm{Wi}$ ); on considère que l'effort horizontal ne doit pas dépasser $1 / 1000^{\mathrm{e}}$ du poids du bateau, ce qui limite donc $i$ à $1 / 1000$, et par conséquent la variation de débit en début et en fin de manœuvre des vannes, qui lui est proportionnelle $(\mathrm{dQ} / \mathrm{dt}=i \times \mathrm{Bgh}$, avec $B=$ largeur du plan d'eau et $H=$ mouillage, soit

$$
\left.d Q / d t<1 / 1000 \times 54 \times 10 \times 4,5 \simeq 2,4 \mathrm{~m}^{3} / \mathrm{s}^{2}\right)
$$

- les vitesses transversales sont à prohiber ;

- les vitesses moyennes longitudinales de courant en bief à proximité de l'écluse (rapport du débit à la section mouillée) ne doivent pas dépasser 0,3 à $0,4 \mathrm{~m} / \mathrm{s}$, afin de ne pas perturber la marche des bateaux: lorsque l'eau de l'éclusée est prélevée ou rejetée en bief, cette contrainte conduit généralement à la détermination du débit maximum pendant l'éclusage (e.g. $60 \mathrm{~m}^{3} / \mathrm{s}$ pour une section mouillée de $175 \mathrm{~m}^{2}$ ) ; si ce débit est par trop insuffisant, en particulier pour les écluses de forte chute, il faut avoir recours, en rivière, à des prises et rejets hors du chenal navigable; en canal artificiel, on est souvent amené à prévoir des bassins d'épargne, comme on va le voir à présent: ces bassins permettent alors d'absorber ou de restituer des débits nettement plus importants en début d'éclusage.

\section{Autres éléments du circuit hydraulique}

Le circuit hydraulique d'une écluse ne se limite en effet pas toujours aux dispositions permettant l'éclusage des bateaux. En canal artificiel, on rencontre souvent des dispositifs permettant d'assurer, de jour, une alimentation en eau du bief aval à partir du bief amont, d'ou nécessité d'une conduite supplémentaire lorsque ces débits sont trop importants pour pouvoir transiter par le sas ou ses propres conduits d'alimentation; de plus, lorsque l'alimentation en eau naturelle du canal est faible, on a généralement recours à des stations de pompage accolées à l'écluse, et à des économiseurs d'eau baptisés "bassins d'épargne", bien décrits dans d'autres exposés.

Signalons enfin, à propos des dispositifs permettant d'économiser l'eau, que la solution des échelles d'écluses (simple, ou double en cas de trafic élevé) reste envisageable, bien qu'elle représente un très sérieux allongement des temps de franchissement (temps de passage des bateaux d'un sas à l'autre en particulier).

\section{Autres évolutions dans la conception des écluses}

Nous avons jusqu'ici insisté sur les tendances actuelles dans la conception des circuits hydrauliques, et sur leurs répercussions pour la conception d'ensemble de l'écluse. II reste à examiner rapidement les autres évolutions dont sont l'objet les écluses :

\section{Les vannes}

Les vannes servent à obturer à volonté les conduites d'alimentation et de vidange de l'écluse. Le problème est d'assurer leur étanchéité, et d'éviter leur mise en vibration, et l'établissement à leur aval de pressions négatives.

On utilise actuellement en France presque uniquement deux types de vannes:

1) Les vannes levantes (à galets, ou, pour les petites vannes, à frottement métal sur métal);

2) Les vannes segment (normales, i.e. à bordé comprimé, ou inverses, i.e. à bordé tendu).

Malgré toutes les précautions prises dans la conception des vannes, celles-ci, très sollicitées, doivent être entretenues ou réparées assez fréquemment sans interruption de la navigation; ceci impose d'avoir deux vannes sur chaque circuit (alimentation et vidange), le batardage de l'une d'elles n'ayant pour effet que d'allonger légèrement la durée des échanges d'eau.

Les vannes des écluses, organes manœuvrés très fréquemment, le sont actuellement presque uniquement par des vérins hydrauliques alimentés par la centrale hydraulique générale de l'écluse ou quelquefois par une centrale propre à chaque tête d'écluse; ces centrales permettent également la manœuvre des portes de l'écluse. 


\section{Les portes}

Les portes d'écluse connaissent une certaines standardisation en France; la porte busquée, qui remonte au moins à Léonard de Vinci, reste la plus employée, en particulier à la tête amont, où elle ne connaît de rivalité que de la part des portes toumantes à axe horizontal, manouvrables en charge, qui permettent donc en principe le passage par l'écluse d'un certain débit lors des crues importantes. A la tête aval, la porte busquée n'est supplantée par d'autres types de portes, en particulier portes levantes, que lorsque la chute est supérieure au tirant d'air de la voie, ce qui permet d'utiliser un mur masque en partie supérieure et une porte de hauteur indépendante de la chute en-dessous.

La présence d'une porte intermédiaire n'est plus retenue dans les projets, du fait du surcoût important qu'elle représente; toutefois, son utilisation se conçoit parfaitement pour une écluse clé, ou présentant des risques, ou pour limiter la consommation en eau sur une voie à grand gabarit et à faible trafic.

\section{Les équipements}

Il a déjà été question de la mécanisation des écluses ; les écluses a grand gabarit comportent en outre des automatismes partiels qui permettent, à partir de la commande par l'éclusier, l'enchaînement des séquences constituant une bassinée ou une fausse bassinée. On peut aller plus loin, en automatisant complètement le fonctionnement de l'écluse, dont la surveillance n'est plus assurée que d'un poste éloigné ; cela n'a pas été tenté sur les écluses à grand gabarit (problème de tailles de bateaux différentes), mais fonctionne couramment sur les écluses Freycinet, allégeant ainsi le coût d'exploitation des voies les plus importantes.

Les autres équipements des écluses ont également connu de grands progrès au cours des années récentes: citons en particulier (et en désordre) les bollards flottants, les circuits fermés de télévision, l'éclairage des sas, la qualité des systèmes de peinture, la signalisation fluviale, les pare-chocs aux portes d'écluses, les batardeaux d'exploitation...

\section{Le génie civil}

Pour le génie civil de l'écluse, déjà évoqué précédemment, nous rappellerons seulement qu'il existe trois grandes catégories de structures de sas :

- les bajoyers autostables (ou bajoyers poids), en béton ordinaire ou faiblement armé, avec radier indépendant, - les structures en $U$ en béton armé ;

- les bajoyers en palplanches métalliques (protégées des chocs par un parement en béton armé) ou en parois moulées préfabriquées, avec ou sans tirants.

Signalons aussi a'ce propos les possibilités d'allongement d'écluses existantes, soit à l'occasion de chômages, soit par préfabrication (d'éléments assemblés sur place par précontrainte, ou de l'ensemble de la partie allongée, coulée, a p̀roximité de l'écluse, et déplacée par flottaison) ou encore par utilisation de palplanches métalliques (si l'alimentation en eau se fait par les portes).

Ces idées assez générales sur la conception actuelle des circuits hydrauliques d'écluses ont surtout voulu montrer les fortes répercussions qu'elle engendre quant à la conception du génie civil de l'écluse. 